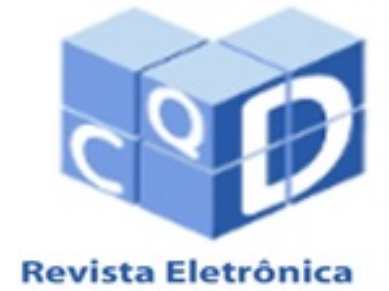

Paulista de Matemática

ISSN 2316-9664

Volume 11, dez. 2017

Gabriela Vicentini de Oliveira

Departamento de Matemática

Aplicada, Imecc, Unicamp

gabriela.voliveira@gmail.com

Edmundo Capelas de Oliveira

Departamento de Matemática

Aplicada, Imecc, Unicamp

capelas@ime.unicamp.br

\section{Sobre a área de polígonos convexos}

On the area of convex polygons

Calcula-se a área de um polígono convexo de $\ell$-lados. Mostrase a expressão que fornece a área de um quadrilátero convexo, devida a Bretschneider, a qual generaliza a clássica fórmula de Brahmagupta, para quadriláteros cíclicos, bem como recupera-se a fórmula de Heron, válida para calcular a área de triângulos. Enfim, justifica-se que o cálculo da área de um polígono convexo de $\ell$-lados, pode ser dado como uma particular soma de áreas envolvendo somente áreas de triângulos, ou somente áreas de quadriláteros ou áreas de triângulos e quadriláteros, dependendo do número de lados do polígono ser um número par ou um número ímpar.

Palavras-chave: Polígonos convexos. Quadriláteros. Fórmula de Bretschneider. Fórmula de Brahmagupta. Fórmula de Heron.

\begin{abstract}
The area of a convex polygon of $\ell$-sides is calculated. It shows the expression that provides the area of a convex quadrilateral, due to Bretschneider, which generalizes Brahmagupta's classic formula, for cyclic quadrilateral, as well as recovering Heron's formula, valid to calculate the area of triangles. Finally, it is justified that the calculation of the area of a convex polygon of $\ell$-sides, can be given as a particular sum of areas involving only triangle areas, or only quadrilateral areas or triangle and quadrilateral areas, depending on whether the number of sides of the polygon is an even number or an odd number.
\end{abstract}

Keywords: Convex polygons. Quadrilaterals. Bretschneider's formula. Brahmagupta's formula. Heron's formula. 


\section{Introdução}

O problema de determinar uma área, que podemos associar ao cálculo de uma integral, é muito mais antigo que a determinação da reta tangente num ponto de uma curva, que podemos associar ao cálculo de uma derivada; os dois pilares do que é conhecido, após Newton e Leibniz, independentemente, com o nome de cálculo diferencial e integral, ou simplesmente, cálculo (CALHEIROS, 2016).

Efetuar o cálculo de uma área, com as ferramentas computacionais que temos hoje, certamente, não parece um problema dos mais difíceis. Ora, por que, então, voltar ao estudo do cálculo de uma área? A matemática se preza pela beleza de uma demonstração ou de uma expressão, que podem traduzir algo abstrato numa linguagem mais, digamos, compreensiva. Um exemplo interessante é o cálculo da área de um círculo, uma figura fácil de se visualizar, porém para chegarmos à bela e simples expressão, $\pi$ vezes raio ao quadrado, que fornece a área desse círculo, se faz necessário o uso do conceito de integral, conceito esse associado a uma soma infinita e, portanto, pode ser considerado abstrato.

Não estamos interessados no cálculo da área de uma figura qualquer, em geral, através de uma integral. O nosso interesse é o cálculo da área de um polígono, ainda mais, um polígono convexo de $\ell$-lados, pois este cálculo nos permite obter uma bela expressão para o cálculo de sua área. Convém ressaltar que a área de várias figuras conhecidas, dentre elas, triângulo e quadriláteros particulares, isto é, quadrado, retângulo, paralelogramo, losango e trapézio, têm expressões simples que fornecem a respectiva área. Mas, para um quadrilátero qualquer, o que devemos fazer para calcular a respectiva área é, por exemplo, dividí-lo em dois triângulos e somar a área desses triângulos de modo a obter a área do quadrilátero. Em geral, dividí-lo em figuras que se sabe como calcular a respectiva área, ou seja, os mencionados acima e dependendo da conveniência, isto é, por exemplo, um triângulo e um quadrilátero ou uma combinação desses dois polígonos.

Existem vários artigos e livros que tratam do tema cálculo de áreas. Mencionamos alguns clássicos, a fim de que se tornem ponto de partida para um eventual aprofundamento no tema. Iniciamos com o artigo de Dostor (1868) e o livro de Hobson (1891), dedicados às construções geométricas e ao cálculo de áreas. Vamos agora nos restringir aqueles mais recentes e de acesso facilitado. Começamos com o artigo de Coolidge (1939) onde o autor discute a expressão da área de um quadrilátero, expressa em termos de seus lados e de dois ângulos opostos, e recupera um interessante resultado, envolvendo os lados de um quadrilátero, devido a Ptolomeu. No artigo de Ivanov, Pinzka e Lipman (1960) encontra-se o problema envolvendo a fórmula de Bretschneider, proposto por Ivanov e as demonstrações, elaboradas por Pinzka e Lipman, do cálculo da área de um quadrilátero expressa em termos dos lados e das diagonais desse quadrilátero.

Aqui, fazemos uma releitura da expressão obtida por Bretschneider a qual fornece a área de um quadrilátero convexo expressa em termos dos quatro lados e de dois ângulos internos opostos, mencionamos também a mesma expressão obtida em função dos lados e das diagonais. Essa expressão, no caso em que temos um quadrilátero cíclico, também conhecido como inscritível, isto é, com os vértices pertencentes a uma mesma circunferência, nos leva a expressão obtida por Brahmagupta que, por sua vez, generaliza a conhecida fórmula de Heron que expressa a área de um triângulo em termos de seus lados e do semiperímetro. Conclui-se mostrando que a área de um polígono convexo de $\ell$-lados pode ser obtida a partir de uma particular soma de áreas envolvendo somente triângulos, ou somente quadriláteros ou triângulos e quadriláteros, dependendo do número de lados do polígono ser um número par ou um número ímpar.

O trabalho está disposto da seguinte maneira: na segunda seção apresentamos a demonstração 
da chamada fórmula de Bretschneider, na terceira seção recuperamos as expressões de Brahmagupta e Heron para, na quarta seção, discutirmos a área de um polígono de $\ell$-lados como soma de particulares áreas envolvendo triângulos e quadriláteros. Observações finais concluem o trabalho.

\section{Fórmula de Bretschneider}

A área de um triângulo pode ser expressa em termos dos lados e do semiperímetro, a conhecida fórmula de Heron, que vamos recuperar na próxima seção como sendo um caso particular da chamada fórmula de Brahmagupta. A fórmula de Brahmagupta fornece a área de um quadrilátero cíclico, isto é, tendo os vértices como pontos de uma mesma circunferência, ou ainda um quadrilátero inscritível, fato esse que não foi mencionado por Brahmagupta. Em 1842, Bretschneider obteve a expressão geral, isto é, a área de um quadrilátero convexo, escrevendo-a em termos dos lados, do semiperímetro e do cosseno da semisoma de dois ângulos opostos.

\section{1 Área de um quadrilátero convexo}

Sejam $a, b, c$ e $d$ os lados de um quadrilátero convexo e $A, B, C$ e $D$ os vértices. Denotemos por $p$ o semiperímetro, isto é, $2 p=a+b+c+d$ e $\alpha$ e $\beta$ os ângulos opostos, conforme Figura 1. Mostremos que a área desse quadrilátero, denotada por $\mathscr{A}$, é dada por

$$
\mathscr{A}_{A B C D}=\sqrt{(p-a)(p-b)(p-c)(p-d)-a b c d \cos ^{2}\left(\frac{\alpha+\beta}{2}\right)} .
$$

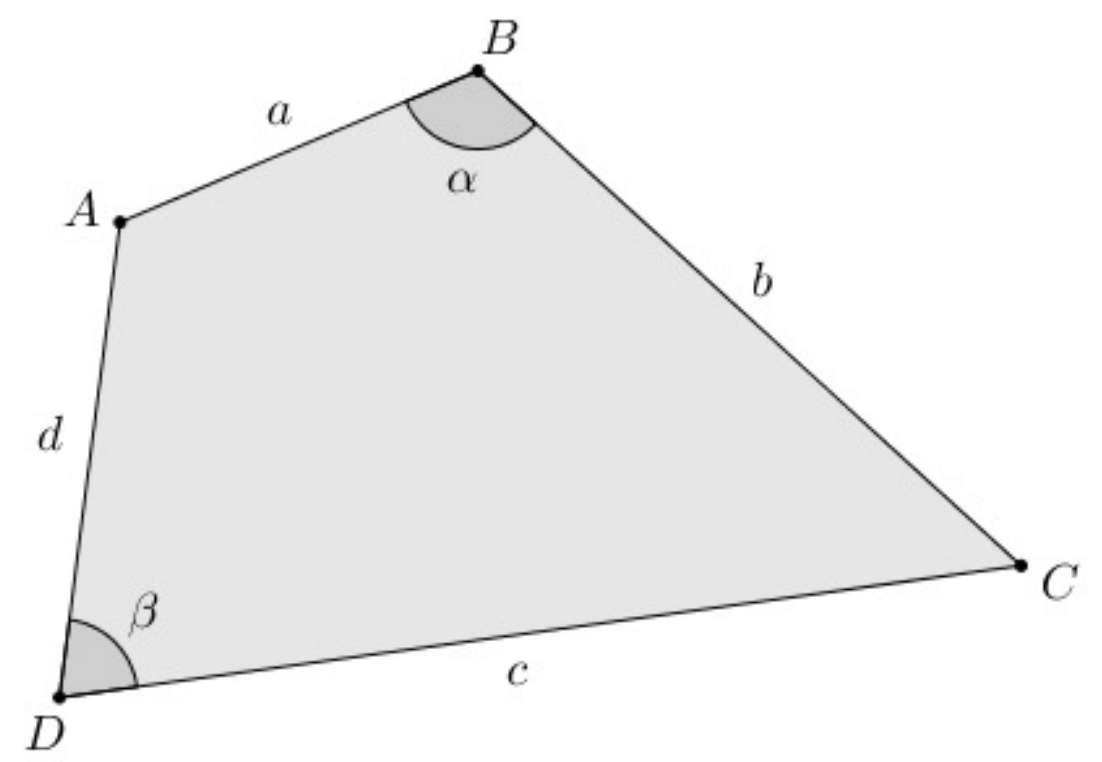

Figura 1: Vértices, lados e dois ângulos internos de um quadrilátero qualquer.

Demonstração. Comecemos pela notação. Sejam $A B=a, B C=b, C D=c, D A=d$, $m(A \widehat{B} C)=\alpha, m(C \widehat{D} A)=\beta$ e sua diagonal $A C=x$, conforme Figura 2. A diagonal $A C$ divide o quadrilátero em dois triângulos, logo a área do quadrilátero pode ser escrita como a soma 
da área dos triângulos,

$$
\mathscr{A}_{A B C D}=\mathscr{A}_{A B C}+\mathscr{A}_{A D C} \quad \Longleftrightarrow \quad \mathscr{A}_{A B C D}=\frac{a b}{2} \operatorname{sen} \alpha+\frac{c d}{2} \operatorname{sen} \beta .
$$

Elevando ao quadrado ambos os membros da expressão anterior obtemos

$$
4\left(\mathscr{A}_{A B C D}\right)^{2}=(a b \operatorname{sen} \alpha)^{2}+(c d \operatorname{sen} \beta)^{2}+2 a b c d \operatorname{sen} \alpha \operatorname{sen} \beta .
$$

Utilizando a lei dos cossenos nos dois triângulos, relativamente à diagonal, e igualando obtemos a identidade

$$
a^{2}+b^{2}-c^{2}-d^{2}=2 a b \cos \alpha-2 c d \cos \beta
$$

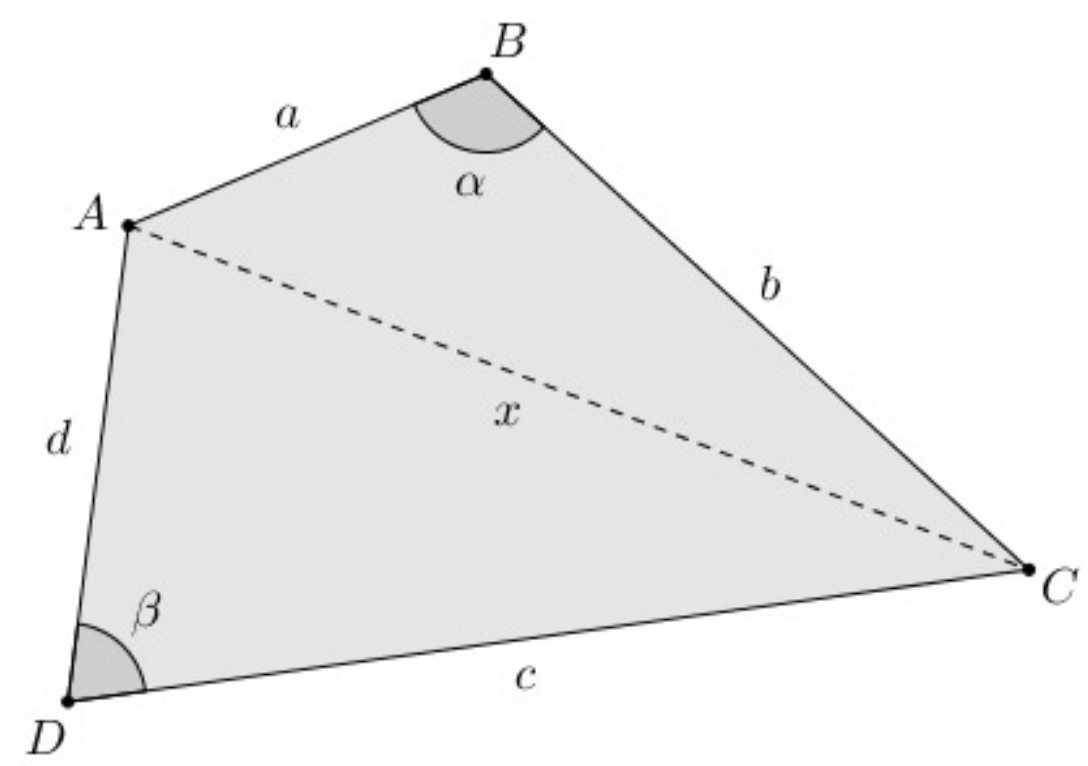

Figura 2: Quadrilátero $A B C D$ e a diagonal $A C=x$.

cujo quadrado permite escrever

$$
\frac{\left(a^{2}+b^{2}-c^{2}-d^{2}\right)^{2}}{4}=(a b \cos \alpha)^{2}-2 a b c d \cos \alpha \cos \beta+(c d \cos \beta)^{2} .
$$

Somamos as Eq.(2) e Eq.(3), utilizando a relação fundamental da trigonometria e fatorando, obtemos

$$
4\left(\mathscr{A}_{A B C D}\right)^{2}+\frac{\left(a^{2}+b^{2}-c^{2}-d^{2}\right)^{2}}{4}=(a b)^{2}-(c d)^{2}-2 a b c d \cos (\alpha+\beta)
$$

onde utilizamos a expressão para o cosseno da soma de dois arcos.

A fim de completar um quadrado perfeito, adicionamos e subtraímos a quantidade $2 a b c d$, bem como utilizamos a expressão para o cosseno do arco dobro e rearranjando temos

$$
4\left(\mathscr{A}_{A B C D}\right)^{2}+\frac{\left(a^{2}+b^{2}-c^{2}-d^{2}\right)^{2}}{4}=(a b+c d)^{2}-4 a b c d \cos ^{2}\left(\frac{\alpha+\beta}{2}\right),
$$

ou ainda, após uma simples fatoração, na seguinte forma

$$
16\left(\mathscr{A}_{A B C D}\right)^{2}=\left[(a+b)^{2}-(c-d)^{2}\right]\left[(c+d)^{2}-(a-b)^{2}\right]-16 a b c d \cos ^{2}\left(\frac{\alpha+\beta}{2}\right) .
$$


Introduzindo o semiperímetro $p=(a+b+c+d) / 2$ na Eq.(6) e rearranjando temos

$$
\left(\mathscr{A}_{A B C D}\right)^{2}=(p-a)(p-b)(p-c)(p-d)-a b c d \cos ^{2}\left(\frac{\alpha+\beta}{2}\right),
$$

ou ainda, visto que a área é um número estritamente positivo, na seguinte forma

$$
\mathscr{A}_{A B C D}=\sqrt{(p-a)(p-b)(p-c)(p-d)-a b c d \cos ^{2}\left(\frac{\alpha+\beta}{2}\right)} .
$$

que é a expressão desejada, isto é, a fórmula de Bretschneider.

Convém ressaltar, como já mencionamos, a fórmula de Bretschneider, Eq.(7), fornece a área de um quadrilátero convexo em termos de seus lados, dos respectivos semiperímetro e dois ângulos opostos, bem como pode ser apresentada em termos dos lados do quadrilátero, dos respectivos semiperímetro e diagonais (IVANOV; PINZKA; LIPMAN, 1960).

\section{Fórmulas de Brahmagupta e Heron}

Nesta seção, como casos particulares da fórmula de Bretschneider, vamos obter as fórmulas de Brahmagupta, relativa à área de um quadrilátero inscritível, bem como a área de um triângulo, ambas expressas em termos dos respectivos lados e semiperímetros.

\subsection{Fórmula de Brahmagupta}

É conveniente lembrar que, uma condição necessária e suficiente a fim de que um quadrilátero seja inscritível, também conhecido como cíclico, é que dois ângulos opostos sejam suplementares. Três diferentes demonstrações da fórmula de Brahmagupta podem ser encontradas em (OLIVEIRA, 2015).

\subsection{1 Área de um quadrilátero cíclico}

Sejam $a, b, c$ e $d$ os lados de um quadrilátero cíclico e $A, B, C$ e $D$ os vértices. Denotemos por $p$ o semiperímetro, isto é, $2 p=a+b+c+d$, conforme Figura 3. Mostre que a área desse quadrilátero, denotada por $\mathscr{A}(\odot)$, é dada por

$$
\mathscr{A}_{A B C D}(\odot)=\sqrt{(p-a)(p-b)(p-c)(p-d)} .
$$

Demonstração. Para um quadrilátero ser cíclico é necessário e suficiente que a soma de dois ângulos opostos seja igual a $\pi$ radianos, isto é, sejam suplementares. Consideremos $\alpha$ e $\beta$ dois ângulos opostos de um quadrilátero cíclico. Temos, então, $\alpha+\beta=\pi$. A partir da Eq.(7) e sabendo que $\cos \pi / 2=0$, segue o resultado.

A expressão dada pela Ex.(8) é conhecida pelo nome de fórmula de Brahmagupta, que expressa a área de um quadrilátero inscritível através dos lados e do semiperímetro.

\subsection{Fórmula de Heron}

Como um outro caso particular da fórmula de Bretschneider, vamos recuperar a clássica fórmula de Heron que expressa a área de um triângulo em termos de seus lados e do semiperímetro. 


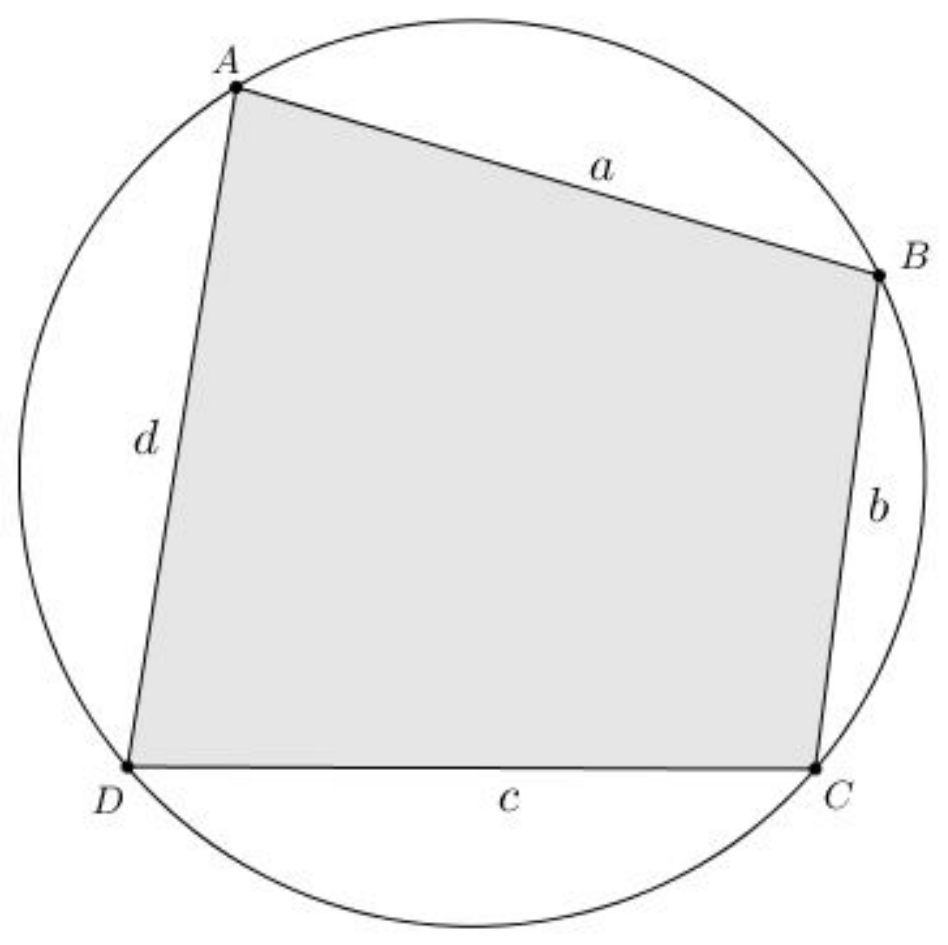

Figura 3: Quadrilátero $A B C D$ inscritível.

\subsection{1 Área de um triângulo}

Sejam $a, b$ e $c$ os lados de um triângulo e $A, B$ e $C$ os vértices. Denotemos por $p$ o semiperímetro, isto é, $2 p=a+b+c$, conforme Figura 4. Mostre que a área desse triângulo, denotada por $\mathscr{A}(T)$, é dada por

$$
\mathscr{A}_{A B C}(T)=\sqrt{p(p-a)(p-b)(p-c)} .
$$

DEMOnstração. Basta considerarmos $d=0$ na fórmula de Bretschneider (ou na fórmula de Brahmagupta) Eq.(7), de onde segue, então, o resultado.

A expressão dada pela Ex.(9) é conhecida pelo nome de fórmula de Heron, que expressa a área de um triângulo através dos lados e do semiperímetro. Convém ressaltar que esse triângulo é qualquer, pois por três vértices de um triângulo passa uma e uma só circunferência o que pode, em princípio, caracterizá-lo como inscritível.

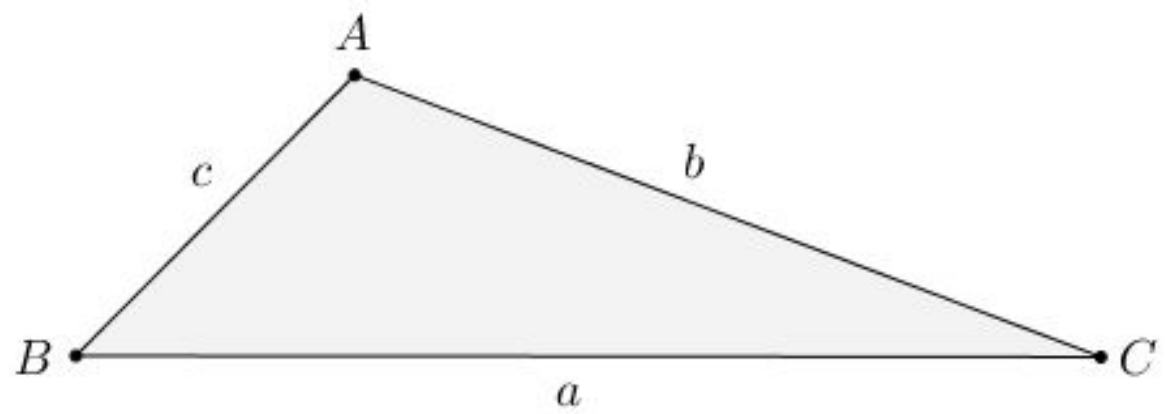

Figura 4: Triângulo $A B C$ qualquer. 


\section{4 Área de um polígono de $\ell$-lados}

Diante do exposto, eventualmente, seria de se esperar que pudéssemos obter uma expressão geral para o cálculo da área de um polígono convexo de $\ell$-lados. Não é esse o caso. Em particular, quando o polígono é regular, sim, podemos escrever uma simples expressão como sendo a soma das áreas de $\ell$ triângulos isósceles.

\section{1 Área de um polígono regular de $\ell$-lados}

Seja $n=3,4, \ldots, \ell$. Sejam $a$ o lado de um polígono regular e $r$ o raio da respectiva circunferência circunscrita. A expressão que fornece a área do polígono regular é dada por (TEIXEIRA; OLIVEIRA, 2016)

$$
\mathscr{A}_{n}=n \cdot \frac{r^{2}}{2} \operatorname{sen}\left(\frac{2 \pi}{n}\right)
$$

onde $2 \pi / n$ é o ângulo do triângulo isósceles oposto ao lado $a$.

Por outro lado, no caso de um polígono convexo qualquer, não temos uma simples expressão que forneça a área desse polígono. Quando muito podemos pensar no cálculo da área desse polígono como sendo uma conveniente soma das áreas de quadriláteros, ou das áreas de triângulos ou uma combinação das áreas de quadriláteros e de triângulos, dependendo de o número de lados ser um número par ou um número ímpar.

\subsection{Polígono com número ímpar de lados}

Para $\ell=3$ a fórmula de Heron fornece a área diretamente. Seja $\ell=5,7,9, \ldots$ o número de lados do polígono convexo. Neste caso, a área do polígono pode ser obtida somando-se as áreas de $5,7,9, \ldots$ triângulos (Heron) ou, alternativamente, o que diminui o número de áreas, $(\ell-3) / 2$ quadriláteros (Bretschneider) e apenas um triângulo (Heron).

\subsection{Polígono com número par de lados}

Para $\ell=4$ a fórmula de Bretschneider fornece a área diretamente. Seja $\ell=6,8,10, \ldots$ o número de lados do polígono convexo. Neste caso, a área do polígono pode ser obtida somando-se as áreas de $6,8,10, \ldots(\ell-2)$ triângulos (Heron) ou, alternativamente, o que diminui o número de áreas, $(\ell-2) / 2$ quadriláteros (Bretschneider).

\section{Conclusões}

Discutimos o cálculo da área de um polígono convexo de $\ell$-lados, demonstrando a fórmula de Bretschneider, que generaliza a clássica fórmula de Brahmagupta, para quadriláreos inscritíveis, e recuperamos a fórmula de Heron, válida para calcular a área de triângulos. Justificamos que o cálculo da área de um polígono convexo de $\ell$-lados, pode ser dado como uma particular soma de áreas envolvendo somente triângulos, ou somente quadriláteros ou triângulos e quadriláteros, dependendo do número de lados do polígono. Enfim, por outro lado, concluímos mencionando que os polígonos circunscritíveis têm a área calculada pela simples expressão $A=p r$, onde $p$ é seu semiperímetro e $r$ o raio da circunferência inscrita. 


\section{Agradecimentos}

GVO é grata à Sociedade Brasileira de Matemática pelo apoio e aos colegas de turma do Profmat.

\section{Referências bibliográficas}

CALHEIROS, J. C. O cálculo com enfoque geométrico. 144 f. Dissertação (Mestrado em Matemática Aplicada e Computacional) - Instituto de Matemática, Estatística e Computação Científica, Universidade Estadual de Campinas, Campinas, 2016.

DOSTOR, G. Proprietés nouvelles du quadrilatère en général, avec application aux quadrilatères inscriptibles, circonscriptibles. Archiv der Mathematik und Physik, Leipzig, v. 48, p. 245-248, 1868.

HOBSON, E. W. A treatise on plane trigonometry. Michigan: University Press, 1891.

COOLIDGE, J. L. A historically interesting formula for the area of a quadrilateral. The American Mathematical Monthly, Menasha, Wis., and Evanston, Ill., v. 46, n. 6, p. 345-347, 1939.

IVANOV, V. F.; PINZKA, C. F.; LIPMAN, J. Bretschneider's formula. The Americam Mathematical Monthly, Menasha, Win., and Buffalo, NY, v. 67, n. 3, p. 291-292, 1960.

OLIVEIRA, G. V. Brahmagupta e quadriláteros cíclicos no ensino médio, $68 \mathrm{f}$. Dissertação (Mestrado Profissional) - Instituto de Matemática, Estatística e Computação Científica, Universidade Estadual de Campinas, Campinas, 2015.

TEIXEIRA, B. H. M.; OLIVEIRA, E. C. Cálculo: exercícios resolvidos para os cursos de exatas e tecnológicas. Campinas: Editora da Unicamp, 2016.

Artigo recebido em jul. 2017 e aceito em nov. 2017. 